\section{》) The new XFELs}

New X-ray free-electron (XFEL) facilities will broaden access to this technology, facilitate methods development, and push boundaries in structural biology.

We first featured XFELs as a Method to Watch in 2011, when this newly established technology promised novel ways of studying macromolecular structure and dynamics. In 2015, we highlighted XFELs' ability to solve high-resolution macromolecular structures from a series of diffraction snapshots of tiny crystals. In 2016, we highlighted the unique abilities of XFELs for capturing very rapid protein structural dynamics.

There are still 'big' reasons why we remain excited about this technology. In 2017, experiments began at the European XFEL in Germany and the Pohang Accelerator Laboratory XFEL (PAL-XFEL) in South Korea. The SwissFEL, located in Switzerland, will begin operating next year. These facilities join the Linac Coherent Light Source (LCLS) in the USA and the SPring-8 Angstrom Compact Free-Electron Laser (SACLA) facility in Japan, which are already in operation.

\section{1) Machine learning in neuroscience}

In the era of big data, neuroscience can profit from deep-learning approaches.

Advances in imaging and recording throughput are generating neuroscience data at an ever-increasing rate, necessitating efficient data analysis approaches. This is particularly evident in subdisciplines such as connectomics, as well as the analysis of behavior or neuronal activity.

Machine-learning and, in particular, deep-learning approaches can help

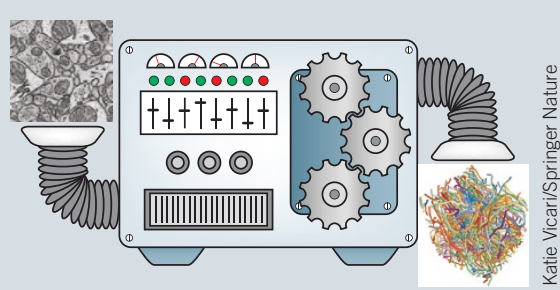

Image processing with machine-learning approaches. Reproduced in part from Dorkenwald, S. et al., Nat. Methods 14, 435442, 2017.
These new XFELs broaden what has been very limited community access to the technology, allowing more researchers to take advantage of XFELs' unique capabilities. The European XFEL in particular has been long anticipated by many in the structural biology community - not only is it the largest facility, but also it is based on new superconducting accelerator technology that enables it to generate 27,000 X-ray flashes per secondmore than 200 times the repetition rate of any other XFEL.

The unprecedented speed and brightness offered by XFELs are crucial for certain types of experiments. XFEL pulses are so short that data can be collected before radiation damage has time to set in; this is particularly important, for example, for resolving highly radiation-sensitive active site structures of metalloproteins. Speed and resolution are also essential for capturing rapid, minute structural changes in enzymes, for example, that are impossible to detect by other methods. Another exciting application is the potential to resolve structures of single biological particles at room temperature, without crystallization, allowing them to be investigated in very close-to-physiological states.

process and analyze large volumes of data. In supervised deep learning, convolutional neural networks learn from training data by passing it through a multilayered network of simple modules. Such networks progressively abstract the data and extract features, which can be applied to analyze new data. Depending on the input data and the way the networks are trained, the extracted features can vary widely.

Image-based data, which are often analyzed manually, can benefit from advanced machine learning. For example, electron microscopic imaging of brain tissue routinely yields terabytes of data that are highly laborious to analyze and annotate manually. Tools such as the SyConn pipeline or the Multicut method (Nat. Methods 14, 435-442, 2017; Nat. Methods 14, 101$102,2017)$ can efficiently segment, and, in the case of SyConn, further analyze such data sets.

Annotating and classifying animal behavior in data sets consisting of hours of video recordings is similarly tedious when conducted manually. Machine-learning

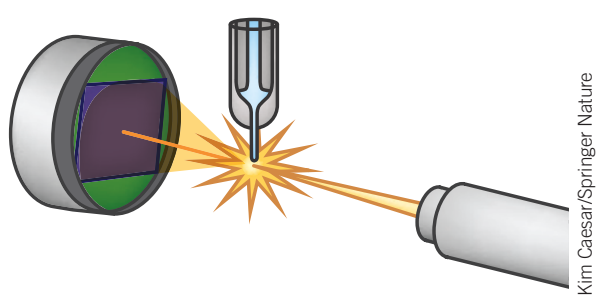

Powerful and ultrafast XFELs enable new approaches to macromolecular structure determination.

The incredible features of the European XFEL should allow researchers to push the realm of discovery in structural biology even further. However, clever scientists need to find new ways to harness this power. The current experimental configurations used at LCLS and SACLA do not allow researchers to take advantage of the high repetition rate of the European XFEL; new approaches for sample delivery, data collection, and data analysis will be needed, especially for realizing single-particle structure analysis at high resolution.

We look forward to seeing what researchers in this field will dream up in their quest to discover new biology.

Allison Doerr

tools such as classifiers as well as more advanced approaches have been applied to analyze, for example, Drosophila or mouse behaviors.

Analyzing calcium-imaging data is another area that has recently seen an influx of machine-learning methods. Such data are notoriously noisy, and extracting neuronal spikes is not trivial, especially since the recorded signal depends on the properties of the calcium indicator used. Supervised learning approaches are flexible enough that they can work on data sets from different calcium indicators than those they trained on.

In select examples, machine learning has proven to be a useful tool in the analysis of the growing deluge of data in neuroscientific research. However, the technology has yet to reach the mainstream. To be adopted by the broader community, machine-learning-based approaches will have to demonstrate their robustness under different conditions. And importantly, efforts will have to be made to facilitate their application and make them easy to use.

Nina Vogt 